\section{Los dispositivos para integrar la docencia con la extensión en la Facultad de Ciencias Médicas de la Universidad Nacional del Litoral}

\section{Yelena Kuttel}

Secretaria de Extensión de la Facultad de Ciencias Médicas. Universidad Nacional del Litoral, Argentina. yelenakutte/@gmail.com

\section{Verónica Reus}

Profesora adjunta de la Facultad de Ciencias Médicas de la Universidad Nacional del Litoral.

vreus@fcm.unl.edu.ar

\author{
María Laura Birri \\ Coordinadora del Programa Equidad \\ en Salud de la Secretaría de Extensión \\ de la Universidad Nacional del Litoral. \\ mbirri@gmail.com
}

Integración de la docencia y la extensión /

Desafíos de gestión

RECEPCIÓN: 24/06/16

ACEPTACIÓN FINAL: 16/10/16

\section{Resumen}

En el Plan de Desarrollo Institucional (2010-2019) de la Universidad Nacional del Litoral concibe a la extensión universitaria como uno de sus pilares fundamentales junto a la docencia y la investigación.

La extensión, como parte sustancial de la vida universitaria, se expresa y materializa en normativas y experiencias concretas. Estas actividades se gestionan a través de diversos dispositivos que garantizan la educación experiencial, entendida como una clase particular de enseñar y de aprender donde los alumnos trabajan junto a los actores sociales para la resolución de temas-problemas reales de la comunidad.

En el presente artículo se relata de qué manera se conjugan la extensión y la docencia en la carrera de Medicina de la Facultad de Ciencias Médicas, tanto desde su diseño curricular en cuanto a Aprendizaje Basado en Problemas del medio social como a través de Prácticas de Extensión de Educación Experiencial, Acciones de Extensión al Territorio y Proyectos de Interés Social.
Palabras-clave

- Docencia y extensión

- Aprendizaje Basado en Problemas

\section{Resumo}

O Plano de Desenvolvimento Institucional (2010-2019) da Universidade Nacional del Litoral concebe a extensão universitária como um dos pilares fundamentais junto com a docência e a pesquisa.

A extensão como parte essencial da vida universitária se expressa e materializa em normas e experiências concretas. Estas atividades se gerem através de diversos dispositivos que garantem a educação experiencial, entendida como uma aula particular de ensino e aprendizado, onde os alunos trabalham junto aos atores sociais para resolver temas-problemas reais da comunidade. Neste artigo se relata de que forma se combinam a extensão e a docência no curso de Medicina da Faculdade de Ciências Médicas, tanto desde o currículo relacionado à Aprendizagem Baseada em Problemas do âmbito social como através de Práticas de Extensão de Educação Experiencial, Ações de Extensão ao Território e Projetos de Interesse Social.

Palavras-chave

- Docência e extensão

- Aprendizagem Baseada em Problemas

\section{Para citación de este artículo}

Kuttel, Y., Birri, M. L. y Reus, V. (2016). Los dispositivos para integrar la docencia con la extensión en la Facultad de Ciencias Médicas de la Universidad Nacional del Litoral. En Revista +E versión digital, (6), pp. 188-193. Santa Fe, Argentina: Ediciones UNL 


\section{La dimensión pedagógica de la extensión}

La extensión es parte sustancial de la vida de la Universidad Nacional del Litoral (UNL). Esto se materializa en normativas y experiencias concretas de actividades que se planifican, gestionan y ejecutan a través de diversos dispositivos institucionales, tales como programas, proyectos, cursos y Prácticas de Extensión de Educación Experiencial. ${ }^{1}$ Estos dispositivos permiten a los alumnos instancias de aprendizaje a partir del análisis y abordaje de situaciones complejas en contexto real, donde se integra el conocimiento académico-científico con otros saberes.

Desde la incorporación curricular de la extensión en el proceso formativo de los estudiantes de las carreras de grado, se procura que los mismos logren asumir una visión solidaria del conocimiento, al mismo tiempo que vivenciar una experiencia enriquecedora para su formación profesional. Así, la Educación Experiencial se constituye en una clase particular de aprender y de enseñar, con un enfoque holístico desde donde relacionar el aprendizaje académico con las situaciones y hechos concretos de la vida real. Es por ello que, como afirma Rafaghelli en el libro Integración docencia y extensión. Otra forma de aprender y de enseñar:

"desde su dimensión pedagógica, la extensión tiene la obligación y el compromiso de crear las oportunidades para que se consolide institucionalmente la formación académica en articulación con las problemáticas sociales”. (2013:36)

Desde sus orígenes, la Facultad de Ciencias Médicas (FCM) de la UNL propone estrategias de enseñanza que tienen como objetivo que el estudiantado logre aprendizajes auténticos. Esto se logra tanto a través del diseño curricular de la carrera de Medicina como a través de otros dispositivos como Acciones de Extensión al
Territorio (AET), Proyectos de Extensión de Interés Social (PEIS), y Prácticas de Extensión de Educación Experiencial (PEEE). Como afirma Camilloni en el libro Integración docencia y extensión. Otra forma de aprender y de enseñar:

"El éxito en el logro de estos propósitos requiere que los currículos y las estrategias de enseñanza sean exitosos en la promoción de aprendizajes auténticos, esto es, aquellos aprendizajes en los que los estudiantes construyen conocimientos 'estratégicos' o 'condicionales' que implican saber por qué, dónde, cuándo y cómo se utilizan esos conocimientos, elaborados, particularmente, en relación con situaciones, casos, problemas o proyectos tal y como se encuentran en la vida real". (2013:11-12)

A partir de valorar la importancia que reviste implicar al estudiantado en la resolución integral de temas-problemas vinculados con la salud en contextos reales, se diseñaron las Unidades de Aprendizaje Basada en Problemas (UBP) como espacios que permiten abordar y resolver situaciones desde una mirada multidisciplinaria y holística. En esta coyuntura los alumnos se constituyen en actores activos, solidarios y autónomos en el proceso de construcción del conocimiento en forma conjunta con otros actores sociales; y los docentes actúan en calidad de guías y colaboradores a la hora de vincular la teoría con la praxis. Tal como expresa Milagros Rafaghelli:

"Estas actividades se consolidan mediante las acciones conjuntas que se tejen en los escenarios sociales/culturales/ históricos con el propósito de comprender e intervenir de manera creativa e innovadora en las situaciones identificadas como problemas sociales". (2013:23)
1) Prácticas de extensión que se desarrollan en el marco de las propuestas curriculares de las carreras de la UNL con el propósito de profundizar alter- nativas de formación de profesionales sólidos y fundamentalmente comprometidos con la sociedad 


\section{4}

\section{el diseño curricular de la carrera de Medicina se sustenta en el Aprendizaje Basado en Problemas del medio social como estrategia pedagógica}

El diseño curricular de la carrera de Medicina de la FCM se sustenta en el Aprendizaje Basado en Problemas (ABP) del medio social como estrategia pedagógica que contribuye a la formación integral de los futuros profesionales desde el razonamiento y el juicio crítico. Esta metodología de enseñanza se implementa durante todo el transcurso de la carrera desde las diversas áreas del plan de estudio que involucran distintas disciplinas. Así, al estudiante se lo implica como protagonista y creador de sus propios aprendizajes al poder desarrollar competencias para el abordaje de temas-problemas relacionados con la salud. Por su parte, el docente actúa como un tutor que ofrece el andamiaje para sostener el autoaprendizaje y la educación continua en diferentes escenarios (centros de salud, centros de cuidado infantil, centros de acción familiar, escuelas, y ONG), para sostener y mejorar la salud pública.

De esta manera, los alumnos tienen la posibilidad de identificar la situación problema, que en muchas ocasiones resulta de la demanda de instituciones y organizaciones sociales y/o del relevamiento previo del futuro escenario de actuación. Esto permite contextualizar los hechos y circunstancias. Seguidamente y con acuerdo de los actores sociales involucrados, elaboran un plan de trabajo con objetivos, actividades, resultados esperados y alternativas de solución a la situación problema. Finalmente las acciones se ejecutan a través de estrategias participativas teniendo en cuenta las características de los destinatarios y sus contextos. Las acciones se analizan permanentemente a través de instancias participativas de coevaluación en los denominados espacios de tutorías, donde según Klug, Jacob y Contini: "se favorece la habilidad del estudiante para trabajar en grupo, respetar objetivos comunes y adquirir un sentido de tarea compartida" (2013:118). Desde esta dimensión se puede trabajar grupalmente con otros actores sociales, articulando lógicas institucionales diversas para el abordaje de diferentes temas.

En este sentido, el ABP representa un claro ejemplo de incorporación curricular de la extensión que facilita y permite a los docentes lograr aprendizajes y evaluaciones auténticas, al poder valorar en los alumnos los saberes y procedimientos de análisis y resolución de situaciones de salud en los distintos contextos (evaluación situada o en contexto). Al mismo tiempo, brinda la posibilidad de implementar una evaluación formativa, definida por Black y William, en el libro Evaluar para aprender, como: "un proceso en el que se recaba información con el fin de revisar y modificar la enseñanza y el aprendizaje en función de las necesidades de los alumnos y las expectativas de logro para alcanzar" (1998:10).

Durante toda la carrera los estudiantes de Medicina tienen la posibilidad de ejecutar prácticas en los centros públicos de salud de la ciudad de Santa Fe y su área metropolitana en el marco de la "rotación de Atención Primaria de Salud". Además, cuentan con la oportunidad de participar en acciones extensionistas y ejecutar prácticas en terreno que responden a las demandas y problemáticas identificadas.

Merece destacarse que a partir del año en curso (2016) los estudiantes pueden realizar la Práctica Final Obligatoria (PFO) en efectores públicos de otras localidades del país bajo la supervisión de un tutor, lo que les permite en muchos casos poder actuar en sus localidades de origen. Desde esta práctica se busca lograr una integración final de conocimientos y habilidades necesarios para abordar los procesos de salud/enfermedad/ atención y resignificarlos al poder realizar acciones en sus propias localidades, enfatizando la promoción de la salud y la prevención de enfermedades prevalentes en Atención Primaria de Salud (APS). Institucionalmente, desde la propia FCM se refuerza año tras año este método de enseñanza con propuestas de Prácticas de Extensión de Educación Experiencial que se implementan a través de distintas áreas curriculares. Desde allí, los alumnos universitarios tienen la posibilidad de trabajar articuladamente con los actores sociales y gubernamentales sobre temas de promoción de la salud y prevención de enfermedades prevalentes.

A su vez, coexisten otros dispositivos como el Sistema Integrado de Programas y Proyectos de la Secretaría de Extensión de la 


\section{al estudiante se lo implica como protagonista y creador de sus propios aprendizajes al poder desarrollar competencias para el abordaje de temas-problemas relacionados con la salud}

Universidad, que habilitan a la participación del estudiantado en proyectos en calidad de voluntarios. Éstos son las Acciones de Extensión al Territorio (AET) y los Proyectos de Extensión de Interés Social (PEIS), que se constituyen en espacios de prácticas y de integración con otras disciplinas.

Estas experiencias enriquecen la formación de los futuros profesionales, ya que contribuyen a valorar el trabajo cooperativo para el abordaje de un problema integrando teorías y metodologías de distintos campos de saber. Al mismo tiempo, es una oportunidad de valorar el trabajo grupal con compromiso, respeto y organización. Sostener y recrear permanentemente un diseño curricular con una fuerte impronta extensionista, al mismo tiempo que generar otros espacios de educación experiencial, es el gran desafío al que invita Camilloni y por el que transita día a día la FCM al

"atreverse a imaginar lo no existente como intento de superación de lo actual, como propuesta de acción, como proyecto a construir en un espacio y un tiempo determinados, contando con ciertas condiciones que lo hacen posible y riesgoso". (2013:8)

\section{Relato de una práctica para la promoción de la salud}

"Conocer para Mejorar la Salud" es una práctica que realizan los alumnos del Ciclo de Promoción de la Salud del segundo año de la carrera de Medicina durante el cursado del "El Ser Humano y su Medio". Se trata de una experiencia de incorporación curricular de la extensión con una duración cuatrimestral. La práctica se viene desarrollando y reeditando a partir del año 2014 bajo un formato de trabajo de dos años consecutivos en un mismo territorio. Así, durante los años lectivos 2014-2015, se trabajó en la localidad de Recreo provincia de Santa Fe, Argentina. Participaron docentes y voluntarios, así como aproximadamente 270 estudiantes cada año distribuidos en 20 comisiones tutoriales.

En dicha práctica se planteó como objetivo integrar y significar los contenidos teóricos abordados durante el ciclo de promoción de la salud. La misma consistió en el relevamiento de determinantes socioambientales, estilos de vida y patologías prevalentes en la comunidad de Recreo, para analizar los aspectos positivos y negativos identificados y pensar líneas de trabajo para futuras propuestas de promoción de la salud y prevención de enfermedades. Los objetivos de aprendizaje fueron: valorar la importancia de la promoción de la salud durante el transcurso de la vida; comprender la relación entre medioambiente y salud; reconocer el campo teórico-práctico de la Atención Primaria de la Salud; resignificar fundamentos teóricos para identificar los problemas de salud de la comunidad. Se coordinaron acciones con el gobierno local, con una ONG y con centros de salud ubicados en la localidad, y se realizaron instancias de capacitación, relevamiento de información, análisis e identificación de situaciones de salud, trabajando con las instituciones participantes para el diseño de propuestas de futuras acciones. En un primer momento se formuló el trabajo extensionista considerando las siguientes premisas:

- Identificar temas/problemas a tratar a través de relevamientos observacionales, encuestas domiciliarias y entrevistas a informantes claves tales como los referentes institucionales de organismos públicos, organizaciones de la sociedad.

- Ser capaces de contextualizar y analizar las características del escenario concebido como el espacio comunitario de la intervención a partir de una investigación de los hechos, datos, circunstancias y área en curso.

- Elaborar una propuesta de trabajo que contenga objetivos, actividades, metodología y recursos.

- Evaluar las experiencias desarrolladas, considerando lo anteriormente planteado con respecto a las estrategias, características de los diferentes escenarios y contextos, entre otros.

Las estrategias de enseñanza de carácter participativo fueron destinadas a estimular la responsabilidad ciudadana.

La práctica en terreno se llevó adelante durante septiembre, octubre y noviembre de 2014 y 2015 y contempló instancias de capacitación 


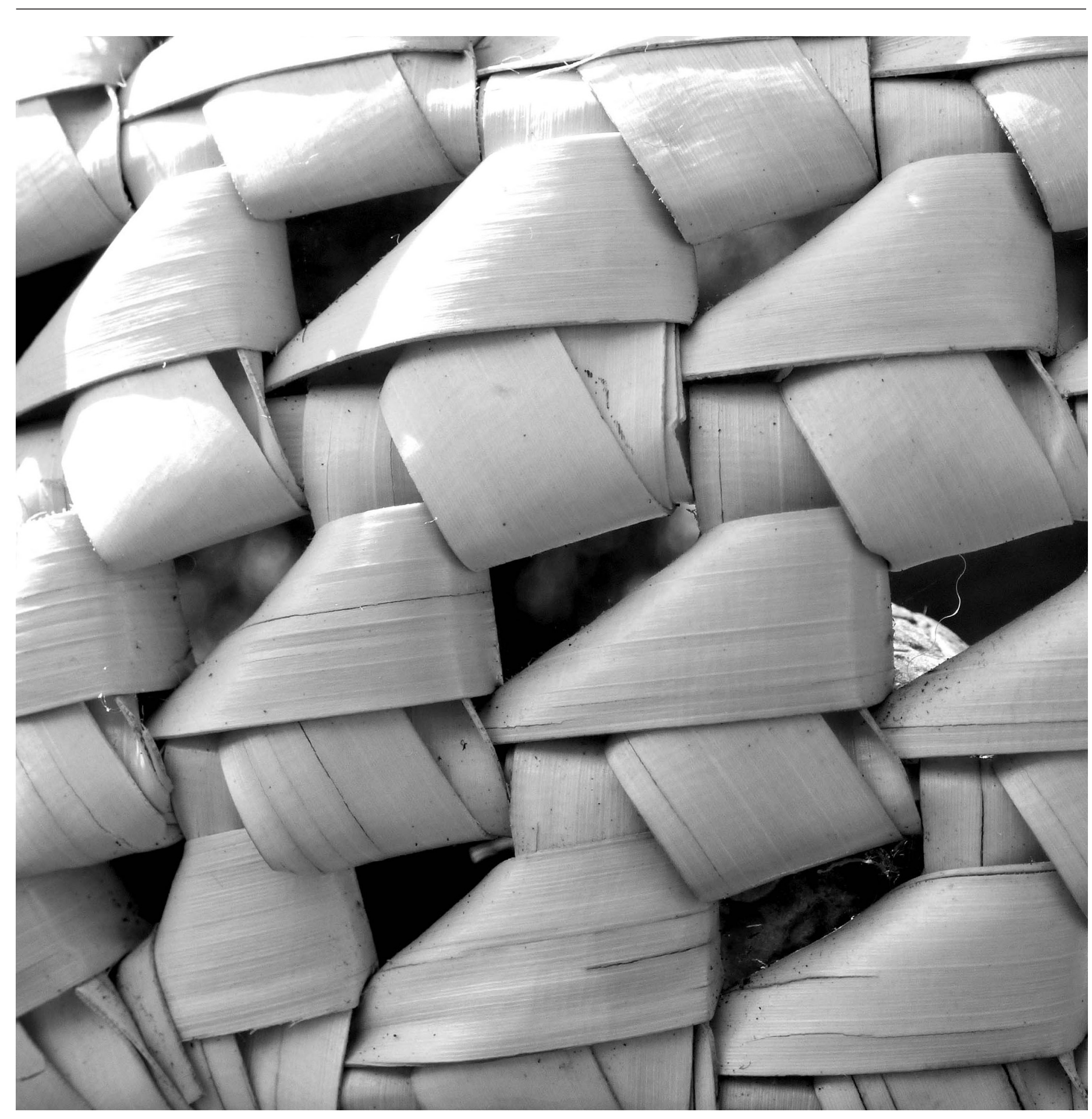

(c) Micaela Block 
y espacios de reflexión de la práctica. Para su realización se coordinaron acciones con la Secretaría de Salud y Medio Ambiente de la Municipalidad de Recreo. A partir de los planos catastrales de la ciudad, se definieron los barrios a relevar, días y horarios para su realización, como también su instrumento.

El relevamiento de determinantes socioambientales se efectuó en forma observacional y las dimensiones trabajadas fueron:

- Accesibilidad en espacios públicos de recreación (plazas, clubes, playones deportivos, biblioteca pública, ciclovías, etc.) y de servicios (Municipalidad, escuelas, centros de salud, etc.).

- Servicios públicos (transporte, cloaca, agua potable, gas natural, estado y seguridad vial, seguridad, recolección de residuos, iluminación pública, mantenimiento de desagües, contención hídrica, etc.).

- Condiciones de las viviendas y peridomicilios.

- Enfermedades prevalentes y comportamientos frente a la salud individual y colectiva.

A partir de los relevamientos, los alumnos realizaron informes grupales que fueron trabajados en diferentes talleres, donde identificaron las situaciones de salud de los distintos barrios y propuestas de trabajo a futuro. Los resultados fueron sociabilizados con las instituciones y organizaciones de la comunidad involucradas a los efectos de acordar futuras estrategias de trabajo en común. Desde estos espacios se logró una integración y construcción conjunta de saberes que enriqueció la formación de los estudiantes universitarios. Para los representantes del gobierno local esto implicó la posibilidad de evaluar sus políticas públicas para poder reforzar o reorientar determinadas acciones.

Cabe destacar que las actividades fueron supervisadas a través de la coordinación académica de $2^{\circ}$ año de la carrera de Medicina. La evaluación continua de aprendizajes fue realizada por tutores guías teniendo en cuenta los desenvolvimientos de los alumnos, la calidad de sus informes individuales y su participación en los talleres de reflexión grupal. Estos últimos encuentros hicieron factible debatir sobre futuras acciones y reflexionar las prácticas profesionales en el contexto social.

La experiencia descripta nos permite ejemplificar, una vez más, cómo desde su gestación el plan de estudio de la carrera de Medicina integra la docencia y la extensión, posibilitando la formación de profesionales médicos comprometidos socialmente, con capacidad de acción en el marco de la salud comunitaria.

\section{Conclusiones}

La propuesta pedagógica innovadora de la carrera de Medicina cuenta con prácticas de extensión incorporadas al curriculum.

Esto refuerza la función social de la Universidad al consolidar los vínculos entre la academia y contexto local inmediato, atendiendo a las necesidades y problemáticas emergentes. Al mismo tiempo, se brinda a los estudiantes la posibilidad de resignificar los contenidos teóricos desde la praxis en tanto se plantean permanentemente nuevos desafíos y adaptaciones ante un mundo en constante cambio, lo que implica estimular en los futuros profesionales una actitud crítica, analítica, reflexiva, creativa, flexible y empática.

\section{Referencias bibliográficas}

Black, P. y William, D. (1998). La evaluación, ¿una instancia para aprender?. En Evaluar para aprender. Conceptos e instrumentos. Buenos Aires: Aique.

Camilloni, A. (2013). La inclusión de la educación experiencial en el currículo universitario. En Menéndez, G. y otros, Integración docencia y extensión. Otra forma de aprender y de enseñar. Santa Fe: Ediciones UNL.

Klug, M.; Jacob, D. y Contini, M. C. (2013). Valoración de una experiencia de enseñanza-aprendizaje en la Facultad de Ciencias Médicas de la UNL. Aula Universitaria, (15), 108-118.

Rafaghelli, M. (2013). La dimensión pedagógica de la extensión. En Menéndez, G. y otros, Integración docencia y extensión. Otra forma de aprender y de enseñar. Santa Fe: Ediciones UNL. 